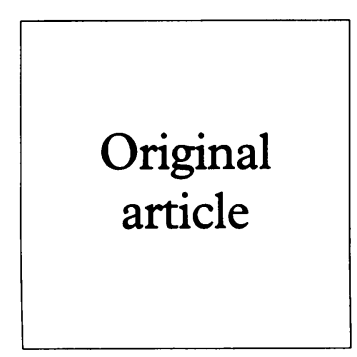

\title{
Pattern of sexually transmitted diseases among pregnant women in Burkina Faso, west Africa: potential for a clinical management based on simple approaches
}

\author{
Nicolas Meda, Lassina Sangaré, Salif Lankoandé, Paul T Sanou, Pierre I Compaoré, \\ Joseph Catraye, Michel Cartoux, Robert B Soudré
}

Objectives: (1) To determine the prevalence of sexually transmitted diseases (STDs) in pregnant women in Burkina Faso. (2) To evaluate the potential of clinical management of STDs based on screening with clinical data and urine leucocyte esterase test (LET).

Methods: Cross sectional study among antenatal clinic attendees was conducted in 1994 in Ouagadougou and Bobo-Dioulasso, the two largest urban centres in Burkina Faso, where more than $94 \%$ of the pregnant women benefit from antenatal care at least twice during their pregnancy. Each woman selected underwent an interview, general and gynaecological examination. Genital samples were collected to confirm the presence of STD pathogens. Logistic regression analysis was done to identify models that predict (a) gonorrhoea and/or chlamydia, (b) trichomoniasis and/or bacterial vaginosis, (c) candidiasis. Sensitivity, specificity and positive and negative predictive values of these models were assessed using standard methods.

Results: All 645 consecutive pregnant women were enrolled in the two sites. Among these women $32.4 \%$ presented at least one STD. The major STDs were: trichomoniasis (14\%), bacterial vaginosis $(13 \%)$, recent syphilis $(3.6 \%)$, chlamydial infection $(3 \cdot 1 \%)$, genital warts $(3 \%)$, gonococcal infection $(1 \cdot 6 \%)$ and genital ulcer $(0 \cdot 8 \%)$. Prevalence of vaginal candidiasis was $14 \%$. The use of a risk marker (length of relationship with regular sexual partner $<3$ years), and the positivity $(+++)$ of the urine LET provided a sensitivity of $80 \%$ and a positive predictive value of $7 \%$ for the screening of gonococcal and/or chlamydial infection. If clinical signs and positivity of the urine LET were taken into account sensitivity and positive predictive value of trichomoniasis and/or bacterial vaginosis screening were $77 \%$ and $37 \%$, respectively. Clinical signs and positivity of the urine LET showed a low sensitivity (23\%) for screening vaginal candidiasis.

Conclusions: The prevalence of STDs in pregnant women is high in urban Burkina Faso. Systematic screening combined with effective treatment should be included in antenatal care in the future. Urine LET, if associated with interview and clinical examination offers a simple, rapid and affordable tool for systematic screening of STDs in pregnant women. However, the proportion of overtreatments with proposed strategies will be high. Further studies are needed to develop and validate better algorithms with probably cheap laboratory tests.

(Genitourin Med 1997;73:188-193)

Keywords: STD; pregnant women; screening; Burkina Faso; Africa

(OCCGE), 01 BP 153

Bobo-Dioulasso 01,

Burkina Faso

N Meda

M Cartoux

National AIDS Control Committee, Ministry of Health, Ouagadougou,

Burkina Faso

$S$ Lankoandé

P T Sanou

Faculty of Health

Sciences, University of

Ouagadougou,

Burkina Faso

L Sangaré

$R$ B Soudré

Department of

Preventive Medicine,

Ministry of Health,

Ouagadougou,

Burkina Faso

I P Compaoré

J Catraye

Correspondence to:

Dr Nicolas Meda, Centre

Dr Nicolas Meda, Centre
MURAZ/OCCGE, 01 BP

MURAZ/OCCGE, 01

153 Bobo-Dioulasso,
Burkina Faso, West Africa.

Burkina Faso, West Africa.

4 December 1996

\section{Introduction}

In developing countries, sexually transmitted diseases (STDs) are a major cause of morbidity and mortality particularly in women and neonates. ${ }^{1}$ Among women of childbearing age, the health problems caused by the STDs include pelvic inflammatory disease, ectopic pregnancy, tubal infertility, carcinoma of the cervix, preterm premature rupture of membranes and postpartum infections. ${ }^{23}$ In neonates, purulent ophthalmia, congenital syphilis, pneumonia, low birth weight and perinatal deaths reflect the most significant consequences of the STDs carried by their mothers. ${ }^{134}$ It is now well documented that the presence of STDs facilitates the acquisition of human immunodeficiency virus (HIV) infection. ${ }^{56}$ For all these reasons the management of the STDs in developing countries is a priority today as part of reproductive health programmes and, moreover, in the campaign against the expansion of HIV epidemic. Among all health interventions to be promoted in developing countries management of STDs may be the most cost effective. ${ }^{7}$

Considering the limited technical and financial resources available in developing countries, the World Health Organisation (WHO) has suggested a syndromic approach based on the complaints of the people who suffer from genital symptoms for STD management. ${ }^{8}$ In some regions, many infected women failed to seek health services only for STD symptoms. ${ }^{9}$ Furthermore STDs in general, Chlamydia trachomatis (CT) and Neisseria gonorrhoeae (NG) infection particularly, are frequently asymptomatic in men $^{10}$ and even more frequently in women. ${ }^{9}$ Several investigations have suggested the limitations of this approach applied to women and have advocated a more active strategy of STD screening at each opportunity of contact by healthcare professionals with a 
woman..$^{91112}$ Pregnancy and antenatal visits constitute privileged moments for STD management in women. The public health impact of this intervention should be considerable for the mother and indirectly for her offspring.

The objectives of this study were to determine the prevalence of different STDs and other reproductive tract infections (RTIs) among pregnant women in Burkina Faso and to evaluate the validity of using sociodemographic parameters, signs and symptoms related to RTIs, and urine leucocyte esterase test (LET) for systematic clinical screening of RTIs during antenatal care.

\section{Methods \\ Population studied and clinical assessment}

This study was conducted among antenatal clinic attendees in 1994 in Ouagadougou and Bobo-Dioulasso, the two largest urban centres in Burkina Faso. Burkina Faso is a landlocked country in west Africa. In 1994 this country had around 10 million inhabitants. The political capital city is Ouagadougou (800 000 inhabitants) which is situated in the middle of the country. Bobo-Dioulasso, situated in the southwest is the economic capital. It has about 350000 inhabitants. The study consisted of cross sectional investigations, enrolling all consecutive pregnant women consulting Saint Camille Maternal and Child Health Centre (MCHC) in Ouagadougou and MCHC of the Caisse Nationale de Securité Sociale in BoboDioulasso between 7 October 1994 and 30 November 1994. These two MCHC are the most important antenatal clinics of these cities in terms of numbers of attendees.

Informed consent was obtained from all participants. A nurse was responsible for administering a standardised questionnaire to each pregnant woman and a physician performed gynaecological examinations including speculum examination and bimanual palpation. Anamnesis included sociodemographic data, previous medical history, sexual behaviour and presence of genital symptoms. As part of the gynaecological examination the physician noted the presence of palpable inguinal lymph nodes, genital ulcers, warts, characteristics of vaginal and cervical discharge if any, and cervical motion tenderness. Vaginal swabs were taken for wet preparation, potassium hydroxide testing and Gram staining. Cervical samples were also taken for Gram staining, NG isolation, and CT detection. The presence of pus on a cervical swab was noted (swab test). Blood and urine samples were also taken.

All RTIs detected in pregnant women were treated free of charge. Syphilis was treated with a single intramuscular dose of 2.4 million IU of benzathine penicillin. Chancroid was treated with a daily oral dose of $2 \mathrm{~g}$ of erythromycin for a period of 15 days. Genital ulcers were treated as syphilis and chancroid. Gonorrhoea and/or chlamydial infection were treated with $2 \mathrm{~g}$ of spectinomycin administered intramuscularly associated with a daily oral dose of $2 \mathrm{~g}$ of erythromycin for 15 days.
Trichomoniasis and/or bacterial vaginosis were treated with a single oral dose of $2 \mathrm{~g}$ of metronidazole. Vaginal candidiasis was treated with topical nystatin. A prescription was given for the treatment of sexual partners. Counselling regarding control of STDs and HIV infection was provided to all pregnant women.

\section{Laboratory assessment}

Vaginal and cervical smears were prepared in sites for direct microscopic examination. Saline wet mounts were examined for the presence of motile Trichomonas vaginalis (TV) and for the presence of pseudohyphae and/or budding yeast cells indicative of Candida albicans (CA) infection. Gram staining smears of vaginal secretions were heat fixed and examined for bacterial vaginosis (BV). The criteria for bacterial vaginosis were the presence of three of the following signs: (1) vaginal fluid $\mathrm{pH}>4.5$, (2) release of a fishy amine odour from vaginal fluid mixed with $10 \% \mathrm{KOH}$, (3) presence of "clue cells", and (4) an abundant flora of predominantly Gram negative bacilli morphologically similar to Gardnerella vaginalis and/or Mobiluncus spp. Material from an endocervical swab was plated onto modified Thayer-Martin medium and incubated in a candle extinction jar at $36^{\circ} \mathrm{C}$ for $24-48 \mathrm{~h}$. Identification of NG was made on the basis of typical colonial morphology, oxidase reaction, and Gram stain result. CT was detected in cervical specimens by an enzyme immunoassay (Microtrak-II, Syva Company, San Jose, CA, USA) and confirmed by a blocking assay using reagents from the same manufacturer. For serological diagnosis of syphilis rapid plasma reagin (RPR) slide test (BectonDickinson, Cockeysville, MD, USA) and Treponema pallidum haemagglutination assay (TPHA) (Fujirebio, Tokyo, Japan) were performed on all blood samples. A positive result with both RPR and TPHA was considered indicative of recent syphilis. A LET was read for colour change after immersion in urine Nephur-Test + Leuco, Boehringer Mannheim GmbH, Mannheim, Germany). The urine LET detects enzymes specific to polymorphonuclear leucocytes (PMNs). The result obtained was semiquantitative and defined by the following pattern: $<10(0)$, $\sim 10-25(+), \sim 75(++), \sim 500(+++)$ PMN $\times 10^{6} / 1$ of urine.

\section{Data analysis}

Frequency of different STDs diagnosed microbiologically was calculated and their $95 \%$ confidence interval (95\% CI) was computed. Categorical variables associated with study site and each STD identified were determined by univariate analysis, using Pearson $\chi^{2}$ test or two tailed Fisher's exact test, as appropriate. Means were compared using analysis of variance or Kruskall-Wallis's test, as appropriate.

Three distinct patterns had been taken into account to elaborate the clinical models for the active screening of RTIs in pregnant women. The first was the presence of NG and/or CT. 
Table 1 Prevalence of reproductive tract infections (RTI) among 645 women attending urban antenatal clinics: Ouagodougou and Bobo-Dioulasso, Burkina Faso, October and November 1994

\begin{tabular}{lccc}
\hline & $\begin{array}{l}\text { Number of women } \\
\text { with infection }\end{array}$ & $\begin{array}{l}\text { Prevalence } \\
(\%)\end{array}$ & $\begin{array}{l}95 \% \text { confidence } \\
\text { interval }\end{array}$ \\
\hline Trichomoniasis & 90 & $14 \cdot 0$ & $11 \cdot 4-16 \cdot 9$ \\
Bacterial vaginosis & 83 & $12 \cdot 9$ & $10 \cdot 4-15 \cdot 7$ \\
Vaginal candidiasis & 90 & $14 \cdot 0$ & $11 \cdot 4-15 \cdot 7$ \\
Gonorrhoea & 10 & $1 \cdot 6$ & $0 \cdot 7-2 \cdot 8$ \\
Chlamydial infection & 20 & $3 \cdot 1$ & $1 \cdot 9-4 \cdot 7$ \\
Genital ulcer & 5 & $0 \cdot 8$ & $0 \cdot 3-1 \cdot 8$ \\
Recent syphilis & 23 & $3 \cdot 6$ & $2 \cdot 3-5 \cdot 3$ \\
Genital warts & 19 & $2 \cdot 9$ & $1 \cdot 8-4 \cdot 6$ \\
Trichomoniasis and/or & 158 & $24 \cdot 5$ & $21 \cdot 2-28 \cdot 0$ \\
bacterial vaginosis & & $4 \cdot 7$ & $3 \cdot 2-6 \cdot 6$ \\
Gonorrhoea and/or & & & $28 \cdot 8-36 \cdot 1$ \\
$\quad$ chlamydial infection & 30 & $32 \cdot 4$ & $38 \cdot 6-46 \cdot 4$ \\
Any STD & 209 & $42 \cdot 5$ & \\
Any RTI & 274 & & \\
\hline A All RTI & & &
\end{tabular}

*All RTI without candidiasis.

†Any RTI = any STD or candidiasis.

The second situation consisted of presence of TV and/or BV. The third situation involved the presence of vulvovaginal candidiasis with CA. Infection with NG/CT were merged for two reasons: (a) they have closely similar symptomatology and complications; (b) the strategies for their prevention and control are also similar. The fact that TV and BV infections have exactly the same treatment explains their combination. The Pearson $\chi^{2}$ test or two tailed Fisher's exact test, as appropriate, were used to compare proportions of infected women with uninfected women in terms of sociodemographic, behavioural, clinical and urine LET data. In each of the three clinical situations, all variables associated with infection at a significant level of $25 \%$ in univariate analysis were selected for stepwise logistic regression analysis.

For the presence of NG/CT infection, the selected variables of the interview data and clinical examination were: age of the women less than 25 years, primigravidae, single or in cohabitating situation, duration of relationship with regular sexual partner of less than three years, recognition of one casual sexual partner during the preceding year, use of modern contraceptive methods in the year preceding pregnancy, to have used condom once at least in life, the presence of yellowish vaginal discharge, to have a positive swab test and a positive urine LET. For the presence of TV/BV infection, the following variables had been selected for multivariate analysis: age of the women more than 34 years, single or in a cohabiting situation, declaration of one casual

Table 2 Distribution of genital symptoms in relation to the presence of selected reproductive tract infections (RTI) among women attending urban antenatal clinics: Ouagadougou and Bobo-Dioulasso, Burkina Faso, October and November 1994

\begin{tabular}{lccc}
\hline$R T I$ groups & No & $\begin{array}{l}\text { Proportion with } \\
\text { vaginal discharge } \\
(\%)\left(95 \% C I^{\star}\right)\end{array}$ & $\begin{array}{l}\text { Proportion with } \\
\text { lower abdominal pain } \\
(\%)\left(95 \% C I^{\star}\right)\end{array}$ \\
\hline $\begin{array}{l}\text { Gonorrhoea and/or } \\
\text { chlamydial infection }\end{array}$ & 30 & $36 \cdot 7(20 \cdot 5-56 \cdot 1)$ & $26 \cdot 7(12 \cdot 9-46 \cdot 2)$ \\
$\begin{array}{l}\text { Trichomoniasis and/or } \\
\text { bacterial vaginosis }\end{array}$ & 158 & $35 \cdot 4(28 \cdot 1-43 \cdot 5)$ & $20 \cdot 3(14 \cdot 4-27 \cdot 5)$ \\
$\begin{array}{l}\text { Vaginal candidiasis } \\
\text { No RTI diagnosed }\end{array}$ & 90 & $28 \cdot 9(20 \cdot 1-39 \cdot 5)$ & $25 \cdot 6(17 \cdot 2-36 \cdot 0)$ \\
\hline *95\% confidence interval. & 367 & $25 \cdot 1(20 \cdot 8-29 \cdot 9)$ & $30 \cdot 8(26 \cdot 2-35 \cdot 8)$ \\
\hline
\end{tabular}

^ $95 \%$ confidence interval. sexual partner in the preceding year, housewife or to have informal business, history of vaginal discharge, to complain of vulvovaginal itching, to show yellowish, or greenish, or homogeneous, or bloody discharge, to have positive swab test and a positive urine LET. For the existence of CA vulvovaginal infection, the following variables were selected: age of the women less than 25 years, primigravidae, claim of one casual sexual partner in the preceding year, to have used condom once at least in the life, to have been treated for vaginal discharge during the preceding year, to $\vec{\Rightarrow}$ complain of vulvovaginal itching, to show ery- $\stackrel{0}{\rightarrow}$ thema of the vulva, yellowish, or greenish, or homogeneous discharge, to have positive swab test and a positive urine LET.

For each defined situation, logistic regression analysis allowed the identification of the variables significantly and independently asso- $\vec{\circ}$ ciated with infection. Significance $(p \leqslant 0.05) \overrightarrow{\vec{\omega}}$ was based on Wald's test. The intrinsic and predictive validity of the three models ? obtained to predict the presence of infection to $\omega$ NG/CT, to observe the TV/BV infection and $\omega$ the presence of vulvovaginal candidiasis were $\vec{\infty}$ finally assessed using standard methods.

\section{Results}

All eligible women agreed to participate in the $\vec{c}$ study. Three hundred and ninety seven pregnant women were recruited in Ouagadougou i and 248 in Bobo-Dioulasso. Pregnant women living in these two urban centres were comparable for age $(p=0 \cdot 17)$, level of education ( $p$ $=0.55$ ), duration of relationship with their $\stackrel{\unrhd}{\varrho}$ regular sexual partner $(\mathrm{p}=0.46)$ and for $\overrightarrow{0}$ STDs prevalence $(p=0 \cdot 12)$. Overall 6453 pregnant women were enrolled. Age ranged from 15 to 41 years [mean 25.3 years, standard deviation (SD): $5 \cdot 9$ years]. Among preg- $\stackrel{\infty}{=}$ nant women, $27 \%$ were primigravidae. Women with at least five pregnancies represented $28.5 \%$ of the study sample. Overall, 60 women $(9 \cdot 3 \%)$ were single, $585(90 \cdot 7 \%)$ lived in a stable relationship with a sexual partner. Among women living in a stable relationship, 93 women (16\%) were in a cohabitating situation and $120(20 \%)$ in polygamous marriage. The mean duration of relationship with their 0 regular sexual partner was 79 months (SD $71 \mathrm{\omega}$ months, range 2-336). Grossly, 33\% of pregnant women knew their present sexual partner for less than three years. The duration of $\mathscr{\mathbb { D }}$ school education in enrolled women ranged from 0 to 17 years, mean $2 \cdot 6$ years (SD 3.8). Eighty three per cent of the women were

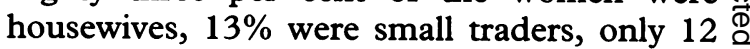
women $(1.9 \%)$ had salaried employment and $16(2 \cdot 5 \%)$ were scholars and/or students. Overall, $60 \%$ of women said they were aware of the condom as a means to control unwanted pregnancies and to avoid HIV transmission. Only $20 \%$ claimed to have used it at least once in their life. Fifteen per cent of women recognised at least one casual sexual partner in the preceding year. Among these women 93\% were married and $7 \%$ were single.

Signs, symptoms or syndromes related to 
Table 3 Potential predictors of selected reproductive tract infection (RTI) among women attending urban antenatal clinics: Ouagadougou and Bobo-Dioulasso, Burkina Faso, October-November 1994

\begin{tabular}{|c|c|c|}
\hline $\begin{array}{l}\text { Variables related } \\
\text { to selected RTI }\end{array}$ & $\begin{array}{l}\% \text { of infected women } \\
\text { (all women }=645 \text { ) }\end{array}$ & $\begin{array}{l}\text { Adjusted odds ratio } \\
\left(95 \% \text { confidence interval) }{ }^{*}\right.\end{array}$ \\
\hline \multicolumn{3}{|c|}{ Gonorrhoea and/or chlamydial infection } \\
\hline Yes & $7 \cdot 3$ & $3 \cdot 1(1.4-6.9)$ \\
\hline No & $2 \cdot 7$ & $1 \cdot 0$ \\
\hline \multicolumn{3}{|l|}{ Urine LET $†$} \\
\hline 0 & $2 \cdot 9$ & $1 \cdot 0$ \\
\hline+ & $6 \cdot 0$ & $1.9(0.7-5.0)$ \\
\hline++ & $4 \cdot 8$ & $1.7(0.5-5 \cdot 7)$ \\
\hline+++ & $8 \cdot 2$ & $2 \cdot 9(1 \cdot 1-7 \cdot 9)$ \\
\hline \multicolumn{3}{|c|}{$\begin{array}{l}\text { Trichomoniasis and/or bacterial vaginosis } \\
\text { Yellowish vaginal discharge }\end{array}$} \\
\hline Yes & $65 \cdot 5$ & $4 \cdot 9(2 \cdot 1-11 \cdot 4)$ \\
\hline No & $22 \cdot 6$ & $1 \cdot 0$ \\
\hline \multicolumn{3}{|l|}{ Urine LET† } \\
\hline 0 & $11 \cdot 4$ & 1.0 \\
\hline+ & $28 \cdot 2$ & $3.0(1 \cdot 8-5 \cdot 0)$ \\
\hline++ & $40 \cdot 9$ & $5.3(3.0-9 \cdot 3)$ \\
\hline+++ & $46 \cdot 9$ & $5 \cdot 9(3 \cdot 5-10 \cdot 3)$ \\
\hline \multicolumn{3}{|c|}{ Vaginal candidiasis } \\
\hline \multicolumn{3}{|c|}{ Vulvovaginal itching } \\
\hline Yes & $23 \cdot 4$ & $1 \cdot 7(1 \cdot 02-2 \cdot 8)$ \\
\hline No & $10 \cdot 7$ & $1 \cdot 0$ \\
\hline \multicolumn{3}{|c|}{ Erythema of the vulva } \\
\hline Yes & $44 \cdot 0$ & $3.5(1.4-8 \cdot 6)$ \\
\hline No & $12 \cdot 7$ & $1 \cdot 0$ \\
\hline \multicolumn{3}{|c|}{ Whitish vaginal discharge } \\
\hline $\begin{array}{l}\text { Yes } \\
\text { No }\end{array}$ & $\begin{array}{l}27 \cdot 3 \\
12 \cdot 1\end{array}$ & $\begin{array}{l}2 \cdot 0(1 \cdot 1-3 \cdot 7) \\
1 \cdot 0\end{array}$ \\
\hline \multicolumn{3}{|l|}{ Urine LET $†$} \\
\hline & $8 \cdot 6$ & $1 \cdot 0$ \\
\hline+ & $12 \cdot 1$ & $1 \cdot 3(0.7-2 \cdot 5)$ \\
\hline++ & $15 \cdot 7$ & $1.9(0.9-4 \cdot 0)$ \\
\hline+++ & $32 \cdot 6$ & $4 \cdot 7(2 \cdot 6-8 \cdot 7)$ \\
\hline
\end{tabular}

${ }^{\star}$ Derived from logistic regression model with variables selected at cut off $p$ value of 0.25 in the univariate analysis.

Urine leucocyte esterase test scoring: $+=10-25 \mathrm{PMNs} \times 10^{6} / 1 ;++=\sim 75 \mathrm{PMNs} \times 10^{6} / 1$; $+++=500 \mathrm{PMNs} \times 10^{6} / 1$.

RTIs (vaginal discharge, vulvovaginal itching, lower abdominal pain, dysuria, genital ulcers, and genital warts) were mentioned by 379 (59\%) women. Clinical examination disclosed signs and/or symptoms of RTIs in 254 $(39.4 \%)$ women. Table 1 shows the prevalence of detected RTIs. Out of 645 enrolled $209(32 \cdot 4 \%)$ had at least one STD, and 274 $(42.5 \%)$ had at least one RTI. Prevalence of RTI associated symptoms according to RTI groups in pregnant women is shown in table 2. Few symptoms were noted. When present, they were non-specific of a given aetiology. Without an identified RTI, $25 \%$ of women complained of leucorrhoea and $31 \%$ complained of lower abdominal pain.

Table 3 gives potential predictors of selected RTIs among pregnant women in Burkina Faso according to multivariate analy-

Table 4 Diagnosis performance of selected reproductive tract infections using sociodemographic, clinical and biological characteristics of 645 women attending urban antenatal clinics: Ouagadougou and Bobo-Dioulasso, Burkina Faso, October-November 1994

\begin{tabular}{lcll}
\hline Performance & $\begin{array}{l}\text { Gonorrhoea and/or } \\
\text { chlamydial infection }\end{array}$ & $\begin{array}{l}\text { Trichomoniasis and/or } \\
\text { bacterial vaginosist }\end{array}$ & $\begin{array}{l}\text { Vaginal } \\
\text { candidiasisł }\end{array}$ \\
\hline No of infected women & 30 & 158 & 90 \\
No of cases diagnosed & 24 & 122 & 21 \\
No of women treated\| & 328 & 330 & 77 \\
Sensitivity (\%) & 80.0 & $57 \cdot 2$ & $23 \cdot 3$ \\
Specificity (\%) & $50 \cdot 6$ & $37 \cdot 0$ & 89.9 \\
Positive predictive value (\%) & $7 \cdot 3$ & 88.6 & 27.3 \\
Negative predictive value (\%) & $98 \cdot 1$ & 87.9
\end{tabular}

*Length of relationship with regular partner $<3$ years and urine leucocyte esterase test (LET) positive (LET $=+++$ )

†Yellowish vaginal discharge and leucocyte esterase dipstick (LED) test positive (LED $\geqslant+$ ).

$\ddagger$ Vulvovaginal itching, erythema of the vulva, and urine leucocyte esterase test (LET) positive (LET $=+++)$.

Number of true cases diagnosed by the clinical screening model built.

\|Number of women treated when the clinical screening model identified was followed (false plus true positives). sis. The duration of relationship with regular sexual partner less than three years and a positivity of at least $(+++)$ with the urine LET were the only variables significantly and independently associated with NG/CT infection in this population. When including the diagnosis of vaginitis ( $\mathrm{TV}, \mathrm{BV}, \mathrm{CA}$ ) in the logistic regression model for NG/CT, LET remained significantly associated with the diagnosis of cervicitis. In this way, vaginitis was not a confounder for the association between LET and cervicitis. Only the presence of yellowish discharge and the positivity of the urine LET were predictive for the TV/BV infection. Finally, four variables were associated with the presence of vulvovaginal CA infection: vulvovaginal pruritus, erythema of the vulva, yellowish vaginal discharge, and once again a positive urine LET

The single variable able to predict any of the three infections groups was a positive urine LET. Table 4 shows the performance of the models chosen for the prenatal screening of NG/CT, TV/BV and CA infections. Overall, these models gave unsatisfactory results particularly for screening of vulvovaginal CA for which only one in four cases would have been identified. However, the sensitivity of the models to screen the NG/CT and TV/BV infections seem satisfactory, with respectively to $80 \%$ and $77 \%$. Should these models have been applied, then most pregnant women would have been overtreated according to low positive predictive value found.

\section{Discussion}

In the present study, we selected women attending antenatal clinics located in the two largest urban centres in Burkina Faso. As 94\% of urban based pregnant women are estimated to attend at least twice the antenatal services during their pregnancy in $1993,{ }^{13}$ we think that these women were representative of pregnant women living in urban and suburban centres in Burkina Faso. In Burkina only $2 \%$ of women of childbearing age are infertile and only $8 \%$ use a modern contraception. ${ }^{13}$ Our report of $42.5 \%$ of pregnant women having at least one RTI and $32 \cdot 4 \%$ having at least one STD emphasises the magnitude of the STD burden in pregnant women in Burkina Faso. The relative importance of each STD varies according, both to the African region, and to different laboratory methods used in the studies. ${ }^{13714}$ Consequently, the polymerase chain reaction or ligase chain reaction for NG and CT diagnosis are now strongly recommended because these methods are more sensitive and specific than NG culture and CT serology used in our study. ${ }^{15}{ }^{16}$ However, independently of these sources of variation many studies, like ours, had reported globally high STD prevalence in Africa. ${ }^{3} 121417$

High STD prevalence in the urban context in Burkina Faso deserves attention and public health interventions for three main reasons. The first is the serious consequences of STDs for mother and child health in terms of infertility, ectopic pregnancy, pregnancy wastage, 
neonatal morbidity and mortality. ${ }^{1-318}$ The high STD prevalence in pregnant women in Burkina Faso explains in part the high incidence of $18 \%$ of low birthweight observed in the country. ${ }^{19}$ The second reason is the high risk of acquisition of HIV infection in STD patients. If we assume the situation found in pregnant women can approximate the situation in the urban population of sexually active adults, this high prevalence of STDs could offer an enormous potential for HIV to spread in Burkina Faso. ${ }^{20}$ The prevalence of HIV infection is already estimated at $8 \%$ in the urban population of Burkina Faso and may further increase owing to the high STD burden if nothing is undertaken to control it ( $S$ Lankoandé, personal communication). The third reason is the opportunity of intervention in the context of the too often neglected antenatal health services in developing countries. Each contact of the health service with pregnant women offers the opportunity to detect health hazards in mothers and babies that should be followed by appropriate management over the whole of the pregnancy. ${ }^{1721}$ In the pregnant population in Burkina Faso STD control should be included as part of antenatal care. Systematic detection of syphilis as part of antenatal care is recommended by WHO. The RPR test is simple and reliable even on the prenatal consultation site and the standard treatment of syphilis is affordable. ${ }^{12}$ The diagnosis of genital ulcers may be made on clinical ground. Treatment should be focused in priority on chancroid and syphilis because of their presence in Burkina Faso and because of the microbiological difficulties in identifying Haemophilus ducreyi. ${ }^{22}$ NG/CT, TB/BV and $\mathrm{CA}$ vulvovaginal infections are particularly difficult to diagnose in pregnant women.

The syndromic approach for the STD management advised by WHO and the national health policy in Burkina Faso are based on complaints (symptoms) and/or signs. Our study suggests that the WHO syndromic approach would miss the chance to treat an important number of pregnant women because STDs are mainly asymptomatic. This situation has already been described by others, ${ }^{91012}$ and supports the importance of adopting an active approach of systematic detection of STDs as part of antenatal visits in pregnant women. The ideal situation for active detection of STDs in pregnant women, a microbiological diagnosis, is often not available in laboratories located in developing countries. Then the use of data from interview, symptoms, clinical signs and complementary examinations during antenatal care, for example, analysis of urine with strips, now constitute the most accessible tools for STD detection in developing countries. The validity of this approach has been tested in our population. The urine LET has been used for the urine analysis. In previous studies this test has shown some utility for identifying correctly patients with NG/CT infection either in male or female patients. ${ }^{2324}$ All the models that we have elaborated by logistic regression analysis for the systematic detection of NG/CT,
TV/BV and CA infection included this simple biochemical test. Overall, clinical screening models gave unsatisfactory results. Our model for detection of NG/CT infection had a low positive predictive value because of the low prevalence of these infections in our study. This situation could involve false treatments for many pregnant women. In our population, the diagnosis of NG/CT without specific laboratory test remains highly problematic. But our model for NG/CT detection had an excel- 3

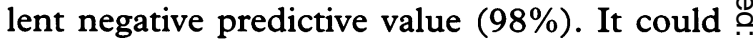
be used to guide positively tested women to a laboratory service, if available, in order to con- $\frac{9}{0}$ firm the diagnosis. Our detection model for TV/BV infection had acceptable sensitivity, $\frac{\bar{c}}{\bar{N}}$ specificity and positive predictive value and $\overparen{\otimes}$ should be used to guide rapid treatment. in Finally our model for the detection of CA infection should not be recommended.

A high prevalence of STDs in pregnant $\vec{\omega}$ women in Burkina Faso have been shown in $\frac{\rho}{0}$ our study. This requires that health workers adopt new active approaches for the systematic detection and management of STDs. A new approach is imperative because of the $\infty$ need to offer pregnant women antenatal care 옥 of quality in order to ensure a favourable pregnancy outcome. It is also integrated with other interventions developed to reduce the spread $\Phi$ of HIV infection in developing countries. But $\vec{\oplus}$ unfortunately, previous and our present stud- .> ies have not found simple and valid tools to screen effectively for STDs. ${ }^{912}$ Further studies are needed to identify and develop alternative clinical screening models. Perhaps future studies should be oriented to the assessment of cheap, simple, and reliable laboratory tests for the diagnosis of RTIs to be used among pregnant women in developing countries.

We thank Drs Jean Gabriel Ouango and Mounio Ouedraogo (National AIDS Control Committee, Ouagadougou, Burkina Faso) for their support and advice. We thank Drs Olga Sankara, Souleymane Zan and Célestin Ouedraogo as well as the team of all the laboratory technicians and midwives. Without their help this study could not have been performed. Special thanks as well are due to Arlette Simonon and Dr Dioulasso, Burkina Faso) for their comments on the manuscript. Nicolas Meda's participation in this work was supported by a grant from ORSTOM.

Financial support of this study was provided by the Ministry of Health, Burkina Faso, with the valuable assistance of consultants from the World Bank (R Paradis, E Van Dyck, M Kamenga).

1 Piot P, Tezzo R. The epidemiology of HIV and other sexually transmitted infections in the developing world. Scand f Infect Dis 1990;69:89-97.

2 Hay PE, Lamont RF, Taylor-Robinson D, Morgan DJ, Ison C, Pearson J. Abnormal bacterial colonisation of the genital tract and subsequent preterm delivery and late miscarriage. BMf 1994;308:295-8.

3 Mabey D, Richens J. Sexually transmitted diseases (excluding HIV). In: Cook GC, ed Manson's Tropical Diseases. 20th HIV. In: Cook GC, ed Manson's Irop

4 Berkowitz G, Papiernik E. Epidemiology of preterm birth. Epidemiol Rev 1993;15:414-43.

5 Kreiss J, Caraël M, Meheus A. Role of sexually transmitted diseases in transmitting of human immunodeficiency virus. Genitourin Med 1988;64:1-2.

6 Grosskurth H, Mosha F, Todd J, Senkoro K, Newell J, Klokke A, et al. Impact of improved treatment of sexually transmitted diseases on HIV infection in rural Tanzania: randomised controlled trial. Lancet 1995;346:530-6.

7 Over M, Piot P. Health sector priorities review: HIV infection and other sexually transmitted diseases. In: Jamisson DT, Mosley WH, ed. Disease Control Priorities in Developing Countries. New York: Oxford University Press, 1993.

8 Organisation Mondiale de la Santé. Prise en charge des patients atteints de maladies sexuellement transmissibles:
OMS, 1991:p112. 
9 Vuylsteke B, Laga M, Alary M, Gerniers MM, Lebughe JP, Nzila $\mathrm{N}$, et al. Clinical algorithms for the screening of women for gonococcal and chlamydial infection: evaluation of pregnant women and prostitutes in Zaire. Clin Infec Dis 1993;17:82-8.

10 Grosskurth H, Plummer F, Mhalu F, Mabey D. STD research in Africa. Lancet 1993;342:1415-6.

11 Braddick MR, Ndinya-Achola JO, Mirza NB, Plummer AF, Irungu G, Sirei SKA, et al. Towards developing a diagnostic algorithm for Chlamydia trachomatis and Neisseria gonorrhoeae cervicitis in pregnancy. Genitourin Neisseria gonorrho

12 Mayaud P, Grosskurth H, Changalucha J, Todd B, West $\mathrm{R}$, Gabone $\mathrm{R}$, et al. Risk assessment and other screening options for gonorrhoea and chlamydial infections in women attending rural Tanzanian antenatal clinics. Bull WHO 1995;73:621-30.

13 Konate LD, Sinare T, Seroussi M. Burkina Faso: enquête démographique et de santé, 1993. INSD and DHS, ed. Calverton, Maryland (USA): Macro International Inc, 1994:296.

14 Laga M, Nzila N, Goeman J. The interrelationship of sexually transmitted diseases and HIV infection: implications for the control of both epidemics in Africa. AIDS 1991;5(supp1 1):S55-S63

15 Wong PC, Ho BSW, Egglestone SI, Lewis WHP. Duplex PCR systeme for simultaneous detection of Neisseria gonorrhoeae and Chlamydia trachomatis in clinical specimens. $\mathcal{F}$ Clin Pathol 1995;48:101-4.

16 Lee HH, Chernesky MA, Schachter J, Burczak JD, Andrews WW, Muldoon S, et al. Diagnosis of Chlamydia trachomatis genitourinary infection in women by ligase chain reaction assay of urine. Lancet 1995;345: 213-6.

17 Leroy V, De Clercq A, Ladner J, Bogaerts J, Van de Perre P, Dabis F. Should screening of genital infections be part of antenatal care in areas of high HIV prevalence? A prospective cohort study from Kigali, Rwanda, 1992-1993. Genitourin Med 1995;71:207-11.

18 Kramer MS. Determinants of low birth weight: methodological assessment and meta-analysis. Bull WHO 1987; 65:663-737.

19 Meda N, Soula G, Dabis F, Cousens S, Some A, Mertens T, et al. Facteurs de risque de prématurité et de retard de croissance intra-utérin au Burkina Faso. Rev Epidém et Santé Publ 1995;43:215-24.

20 Chin J. Public health surveillance of AIDS and HIV infections. Bull WHO 1990;68:529-36.

21 Rooney C. Antenatal care and maternal health: how effective is it? a review of the evidence. Geneva: WHO/MSM/92.4, 1992:p74.

22 Ledru S, Meda N, Fofana M, Soula G, Bazie AJ, Chiron $\mathrm{JP}$, et al. Etiologic study of genitourinary infections in women of childbearing age in Bobo-Dioulasso, Burkina Faso, 1992. Sex Transm Dis 1996;23:151-6.

23 Shafer MA, Schachter AJ, Moscicki AB, Weiss A, Shalwitz $\mathrm{J}$, Vaughan $\mathrm{E}$, et al. Urinary leukocyte esterase screening test for asymptomatic chlamydial and gonococcal infections in males. $\mathcal{F} A M A$ 1989;262:2562-6.

24 Lewis J, Kraus S, Lossick J. Use of the urine leukocyte esterase test to screen women for gonorrhea and chlamydia infections. In 94th General Meeting of the American Society for Microbiology. Washington DC, 1994: Abstract C-350.

\section{Historical vignette}

\section{A Cyprian Ode}

Says Venus to Cupid, "It grieves me to think

What ills my dear votaries befall,

Who, whilst my sweet nectar they eagerly drink,

Too oft find it mingled with gall.

What left-handed Demon thus dashes the bowl,

As grudging poor mortals the bliss;

Who sheds the contagion that darts through the

soul,

And kills with a treacherous kiss?

Bid Mercury straight go and bind the fell fiend

In chains adamantine, I pray

That lovers may hence give their fears to the

wind,

And joyful proclaim holiday.

From hence let them banish dull sorrow and care,

Needless torture no longer endure;

Lo! I send down Apollo to impersonate

C - - - e, *

Who shall teach them a lenient cure."

^Peter Clare was a London surgeon. He quoted lines about himself in a book on venereal disease which he edited, published in 1783. "Cyprian" formerly meant "lewd". 